\title{
Submergence of magnetic flux in interaction of sunspot groups
}

\author{
B. Kálmán
}

\author{
Heliophysical Observatory of the Hungarian Academy of Sciences, PO Box 30, Debrecen, Hungary
}

Received 13 December 2000 / Accepted 2 March 2001

\begin{abstract}
Submergence of magnetic flux is demonstrated in the process of evolution of sunspot groups NOAA 6850 (26 Sep.-07 Oct. 1991) and 7220/22 (06-17 July 1992). In both cases new magnetic flux emerges immediately behind an existing spotgroup. The new flux does not interact with the old one, as can be seen in YOHKOH X-ray images, so no significant flare activity occurs, although umbrae of different magnetic polarity collide. In both cases the quickly forward moving $p$-spots of the new flux force the submergence of the $f$-spots of the older region, these being squeezed from two sides between the old and new $p$-spots. This leads to the disappearance of about $1610^{21} \mathrm{Mx}$ of $f$-polarity in $\mathrm{AR}$ 6850. In the case of this region the submerged flux interacts with newly emerging magnetic fields and in the next rotation continues its life as the complex and eruptive AR 6891. In AR 7220/22, spots of both polarities submerge in the intermediate part of the complex between the old leader of AR 7220 and the quickly growing AR 7222, leaving in the next rotation a bipolar-looking group (AR 7248), consisting of two $p$-polarity spots. So, submergence of magnetic flux due to interaction of old and new activity may play an important role in the decay of sunspot groups.
\end{abstract}

Key words. Sun: activity - Sun: magnetic fields - sunspots

\section{Introduction}

The decay of the magnetic flux of sunspots is a serious problem of solar physics since Cowling (1946) demonstrated that their physical parameters (magnetic field, conductivity of the photospheric material) imply an $\sim 300$ year lifetime. As ohmic dissipation cannot be the general cause of the decay of the photospheric magnetic fields, some other mechanisms (reconnection, turbulent erosion, submergence, expansion, ejection) must play a significant role. Magnetic reconnection is believed to be the energy source of flares and also can play an important role in the expansion and ejection of magnetic flux (Priest \& Forbes 2000), although observational evidence on the influence of large flares of the decay of sunspot fields is controversial. The slow erosion of sunspot fields through turbulent convection (Petrovay \& Moreno-Insertis 1997) may be seen best in the case of decay of old, quiet, isolated spots. In evolving complex groups, however, other processes can also be observed. Rabin et al. (1984) described a disappearance of $410^{20} \mathrm{Mx}$ of magnetic flux in 24 hours in the well-studied SMY NOAA Active Region 2372, and attribute this to the submergence of the field. Zirin (1985) showed an example of the appearance and submergence of a small active region in four days. Garcia de la Rosa \& Collados (1987) displayed a case of convergence and submergence of a dipole in NOAA 4588. Wang (1992) observed the cancelling of $410^{21} \mathrm{Mx}$ of flux in two days in

Send offprint requests to: B. Kálmán,

e-mail: kalman@tigris.klte.hu
AR NOAA 6233 in a potential field configuration without large activity, so he also proposed submergence (or emergence) of an U-shaped magnetic loop. Three other cases of flux cancellation he associated with fast reconnection in flares. Harvey et al. (1999) recently demonstrated, comparing photospheric, chromospheric and coronal observations, that in the process of cancellation of network dipoles, the magnetic field also submerges.

Complex sunspot groups are usually more flare-active than simple bipolar ones (Sammis et al. 2000). Especially active are the so-called delta-configuration groups (Kunzel 1960), in which umbrae of opposite polarity share a common penumbra, sometimes in close contact. Although delta spotgroups produce most of the large flares, not all delta configurations are active (Zirin \& Liggett 1987). Studying inactive delta groups may uncover the main differences which are crucial in flare activity. Detailed investigation of the evolution of complex sunspot groups also can give information about the subsurface processes of active region formation and hints on significant and insignificant characteristics of flare-active sunspot groups. The Debrecen Heliophysical Observatory has a unique collection of observational material spanning more than four decades. A longer program of study of complex active regions was begun several years ago.

In this program, the long-term evolution of a complex of activity in the southern hemisphere of the Sun around Carrington longitude 180 between July 1991-April 1992 was studied by Bumba et al. (1996). AR NOAA 6850 (Sep. 26-Oct. 7, 1991) appeared in this complex of 
activity; it was large, magnetically complex and evolving, but was not flare-active, although large motions were observed in it (Bumba et al. 1995). Another active complex, containing regions NOAA 7220/22 and NOAA 7428/51 (July 6-18 and Aug. 02-13 1992) was also selected; in these spotgroups quick growth and large sunspot proper motions also occurred. In both complexes the newly emerging flux emerged on the following (eastern) side of the old activity, and the usual westward drift of new sunspots led to collision and interaction between newly emerging and old sunspots. In this interaction, submergence of substantial amount of existing magnetic flux was observed.

\section{Observations and data reduction}

White-light full-disk observations were obtained in the Debrecen Heliophysical Observatory. Whole-disk observations of $10 \mathrm{~cm}$ solar image diameter were made on highcontrast photographic films; these were measured with $0.01 \mathrm{~mm}$ accuracy on an ASCORECORD measuring machine. The method of observations and their reduction was described in more detail in Bumba et al. (1993, 1995). For the present work the coordinate measurements of the umbral and penumbral boundaries (1500-2000 points on the best photograph of the day) were used, the measurements were corrected for instrumental and atmospheric effects, then transformed into Carrington coordinates. The drawings of the sunspot groups in Figs. 2, 3 are produced from these coordinate measurements, in Carrington coordinates. As both studied regions have a latitude near $12^{\circ}$ South, effects of the solar differential rotation are small. Photographic observations of AR 6850 were also digitized with a SONY video camera, and the images converted to Carrington coordinates, based on ASCORECORD measurements of the same films (Fig. 1).

NSO/Kitt Peak Observatory magnetograms were obtained fron the NSO Internet archives, and registered to the Debrecen photographs by the intensity channel, taking into account the time difference. Magnetograms were also converted to Carrington coordinates (Fig. 1). For AR 6850 there was a magnetogram for every day of the transit of the group except for Sep. 27, but for AR 7220/22 only on 5 days out of 12 .

The coronal images were obtained by the Soft X-ray Telescope (SXT) (Tsuneta et al. 1991) aboard the YOHKOH satellite (Ogawara et al. 1991). The images used in the present study were retrieved from the YOHKOH database at the NASA Goddard Space Flight Center and from the Yohkoh Data Archive Centre at the Mullard Space Science Laboratory of University College London (UK) through the Internet.

On the whole-disk SXT images the solar limb was determined, and an overlay of the Carrington coordinate net and the spot contours (positionally corrected for the time difference) was copied onto them (Figs. 6, 7). The solar limb could be defined within one pixel, so the accuracy of the overlays is about 5 arcsec. Another source of
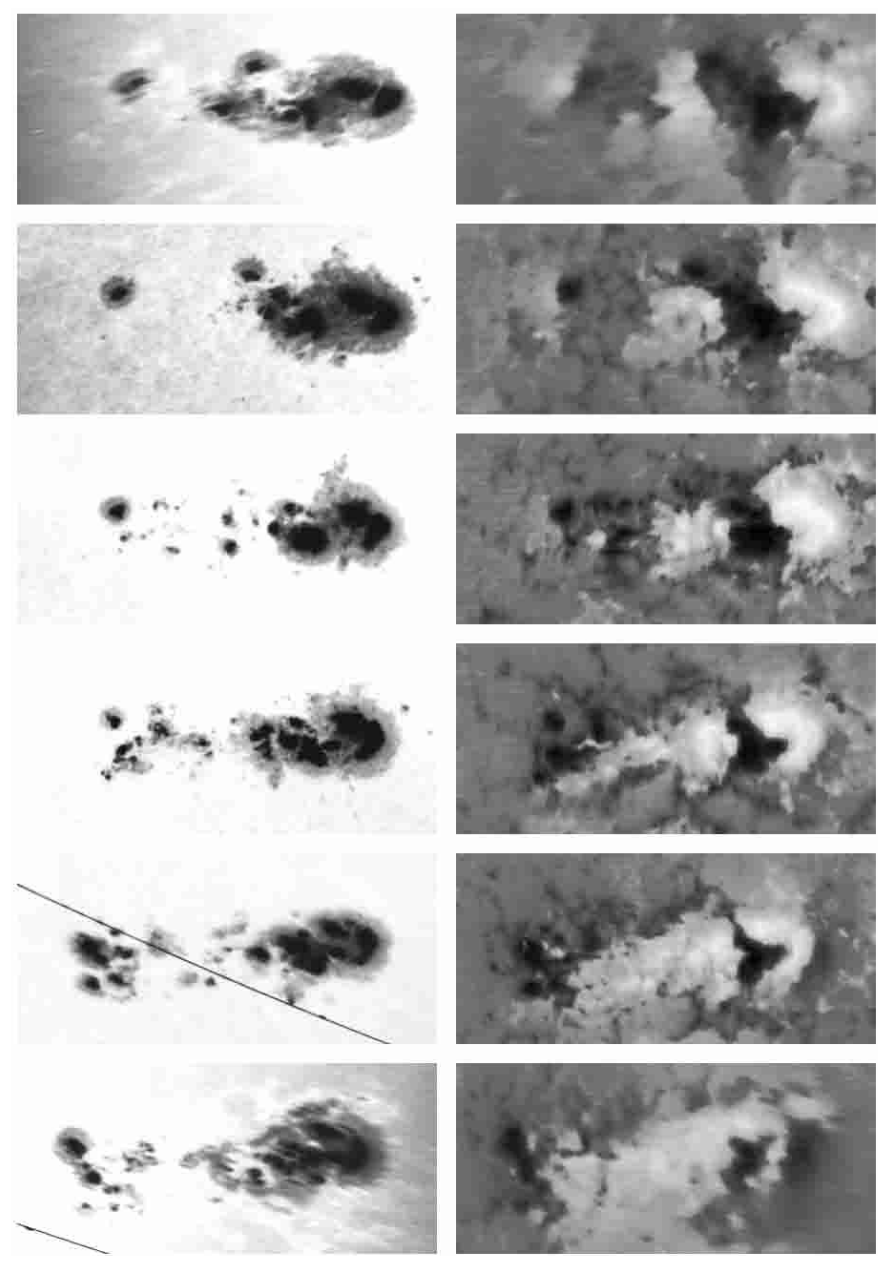

Fig. 1. Rectified photographs (left column) and magnetograms (right column) in Carrington coordinates of AR 6850 for September 26, 28, 30 and October 2, 4, 6, 1991. Photographs from Debrecen Heliophysical Observatory, magnetograms from the Kitt Peak Observatory Internet archives. The submergence of the following (black) polarity can be well followed in the western (right) part of the group (cf. Fig. 2)

misalignment can be the time difference between the SXT and photospheric images, the former are mostly near UT noon, the latter mostly around 6 hours UT for AR $7220 / 22$. Although the positions in the sunspot drawings are corrected for the rotation of the Sun, evolutionary changes and sunspot proper motions cannot be interpolated for the time difference. Taking into account the largest proper motions (Bumba et al. 1995), the resulting displacements can be less than 1-2 arcsec.

\section{Evolution of the sunspot groups}

AR 6850 emerged on the east limb on Sep. 25, 1991 as a delta configuration, with several umbrae in a common large penumbra, and a single follower spot, not counting some small spots around the large penumbra. The group displayed a clear delta-configuration, as in the large penumbra the preceding $(\mathrm{W})$ pair of umbrae was of $p$ polarity, then in the middle a NE-SW oriented chain of 

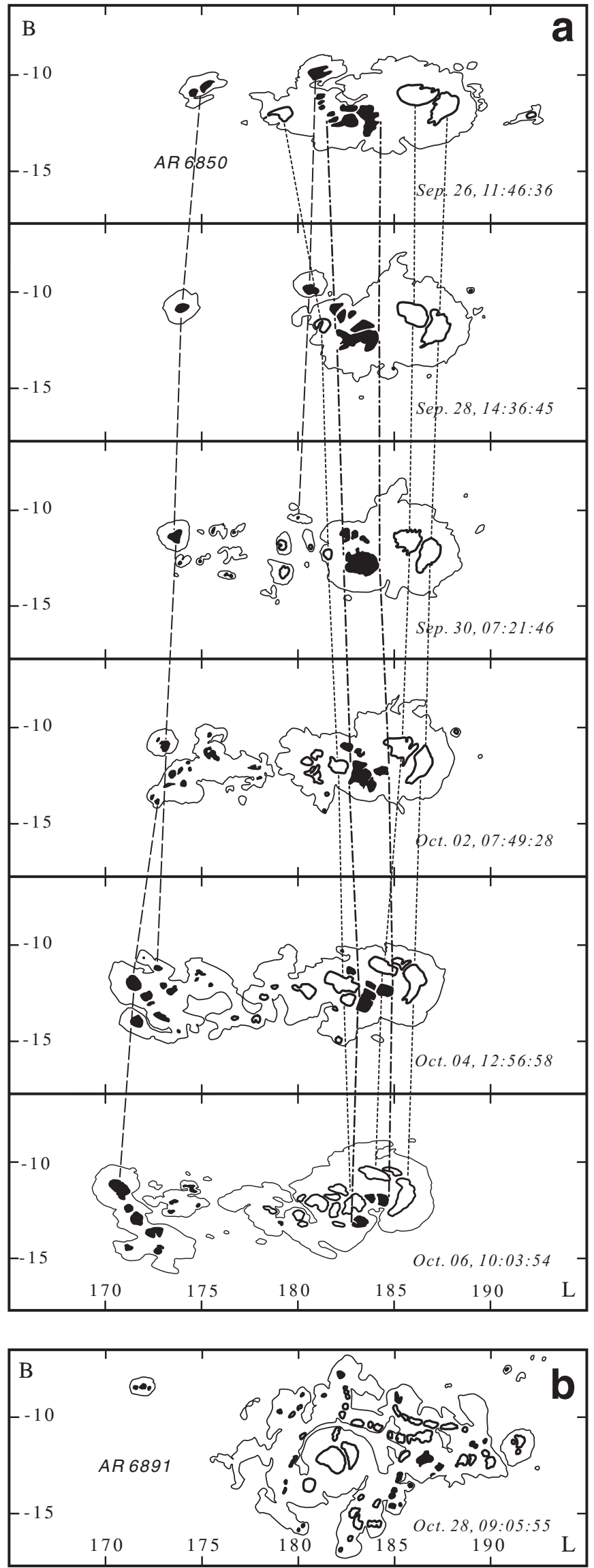

Fig. 2. a) Contour measurements of AR 6850 in Carrington coordinates. Thin line - penumbra, thick line - umbra. Umbrae of $f$-polarity are filled. Positions of some umbrae are connected to emphasize proper motions, dotted line $p$-polarity, dashed line - f-polarity, dot-dashed line marks the submerging $f$-polarity part. b) The same area of the solar photosphere one rotation later around the central meridian, showing complex AR 6891, consisting of the remnant $p$-umbrae, and the surrounding flow of new, N-S oriented magnetic flux 
f-polarity umbrae followed, on the eastern boundary again a new small $p$-polarity umbra was visible.

In the following days the initially distorted $f$-polarity umbra became a regular round one until Sep. 30, but from Sep. 29 new magnetic flux also began to emerge in the middle of the sunspot group as small pores and spots of both polarities. The $p$-spots of the new activity moved vigorously westward, pushing the f-polarity to the leader umbra pair. In this process the regular $f$ umbra became fragmented and reduced in size, the leader umbra pair was deflected to the north in its backward (eastward) drift. The $f$-spots moved eastward, circumventing the single, separate follower spot, which slowly dissolved on Oct. 6. The old f-polarity umbrae became squeezed between the quickly forward moving new $p$ polarity umbrae and the slowly backward moving leader umbra pair (see Figs. 1, 2a). In this process the $p$-polarity leader umbra pair became severely distorted, and the $f$ polarity flux slowly disappeared, seemingly submerged. Interestingly enough, this colliding motion of opposite magnetic polarities did not produce any larger flares. The only 3B/M7.3 large flare on Sep. 29 occurred to the South of the spotgroup, probably due to interaction with the newly emerging AR 6853 (Fig. 6, see Bumba et al. 1995).

In the next rotation this sunspot group appeared as the very active and complex AR 6891 at the eastern limb on Oct. 23. In the center there was a triple $p$-polarity umbra, consisting of the remains of the double leader umbra of AR 6850 plus the newly appearing $p$-polarity, what was surrounded by a westward flow of new, North-South oriented magnetic flux forming a wake on the western side of the old spots (Fig. 2b, see also Kálmán 1997).

The other studied complex contained ARs 7220 and 7222 (Fig. 3a). When it appeared at the eastern limb (July 6, 1992), only AR 7220 was visible, a seemingly fairly simple bipolar group. From the other spotgroup (AR 7222) only a small $p$-polarity spot was seen. Looking closer at AR 7220, from the distribution of polarities and proper motions of umbrae it became clear that the leader spot was independent and older (it had the characteristic eastward drift and regular shape of the old $p$-spots, and was of noticeable distance from the rest in the first days). The remaining part of AR 7220 formed a compact bipolar group with a poorly developed, distorted $p$-polarity part. In the first days the divergent motion of $p$ - and $f$-polarity umbrae was observable, but as this bipolar group was compressed from both sides (between the slowly backwards drifting leader and the quickly evolving $p$-part of AR 7222), the prevalent direction of motion changed, and umbrae begin to move generally southwards. They also shrank and submerged, so when the group disappeared behind the western limb, only the quiet, almost unchanged leader spot remained. Flare activity of this group was moderate until July 10, with 10-13 subflares and 2 Imp 1 flares per day (in X-rays mostly $\mathrm{C}$ class and 1-1 M flares per day), the largest events were a 1N/X1.2 flare on July 8 and a 2N/M6.7 event on July 9. As the area of the compact
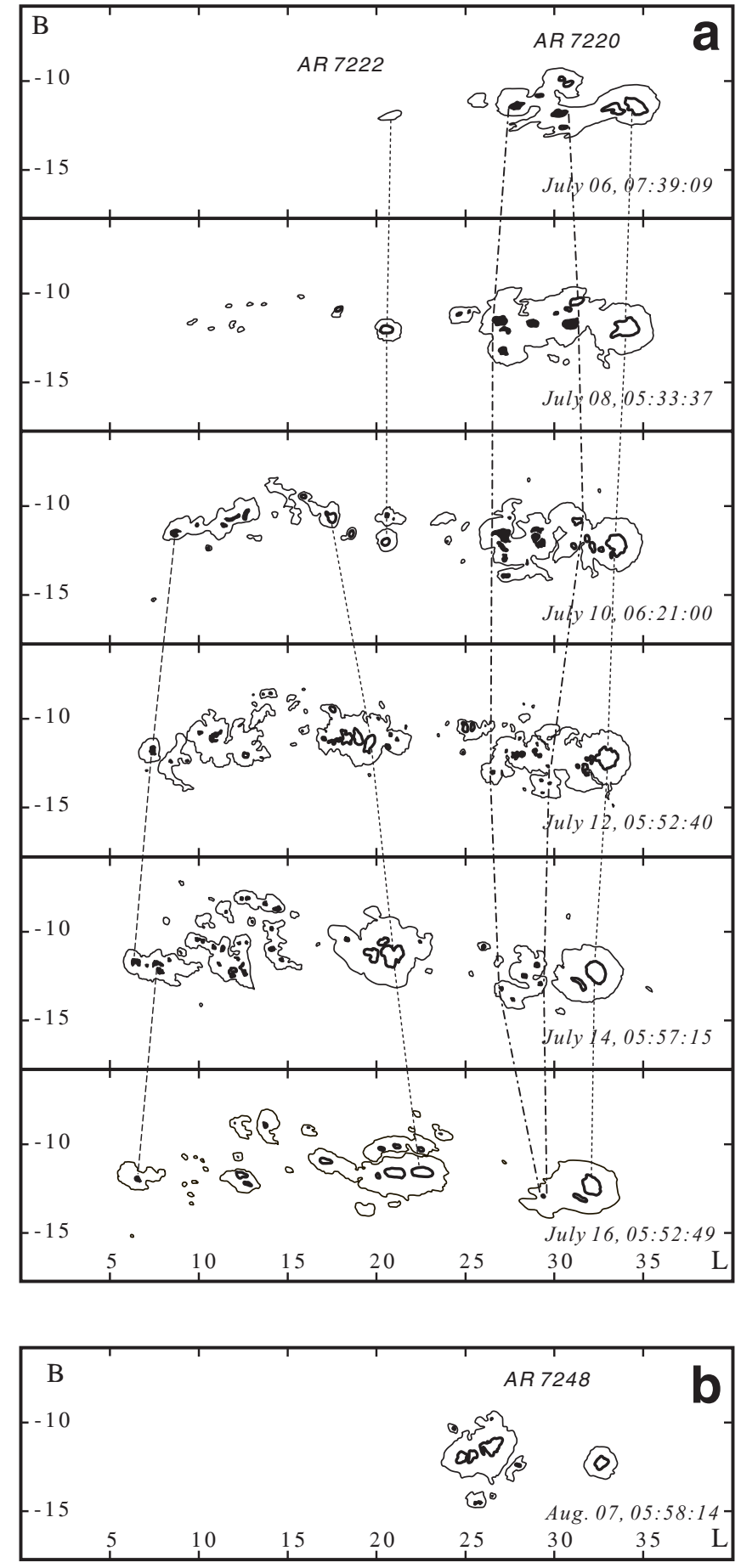

Fig. 3. a) Contour measurements of $\mathrm{AR} 7220 / 22$, using the same conventions as in Fig. 2. b) The same area of the solar photosphere at central meridian passage in the next Carrington rotation

part decreased, from July 11 the flare activity decreased to 5-6 subflares of $\mathrm{C}$ class per day.

The firstly appearing $p$-polarity spot of AR 7222 did not show the quick westward drift typical of its polarity. As can be seen on the coronal images (Fig. 7a), this spot interacted more with the $f$-polarity spots at the eastern edge of AR 7220. The quick evolution in AR 7222 began on July 10, with significant growth and divergence of 


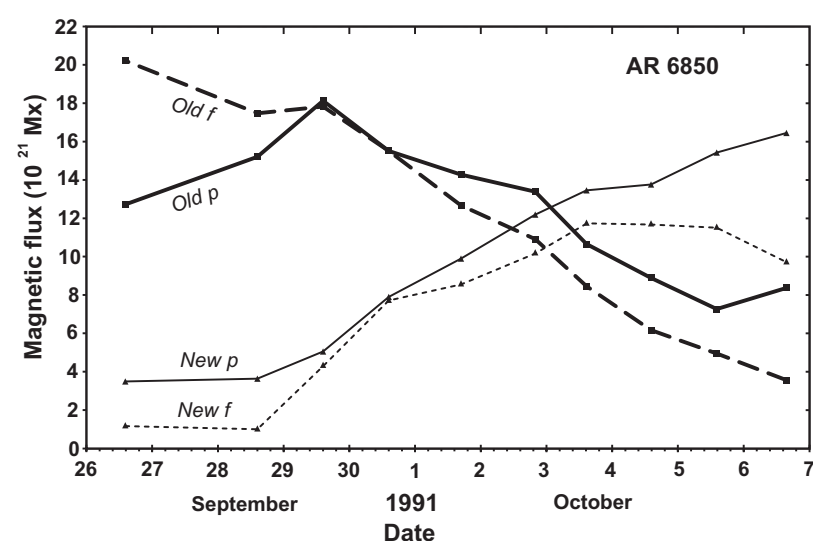

Fig. 4. The changes of magnetic flux in various parts of AR 6850

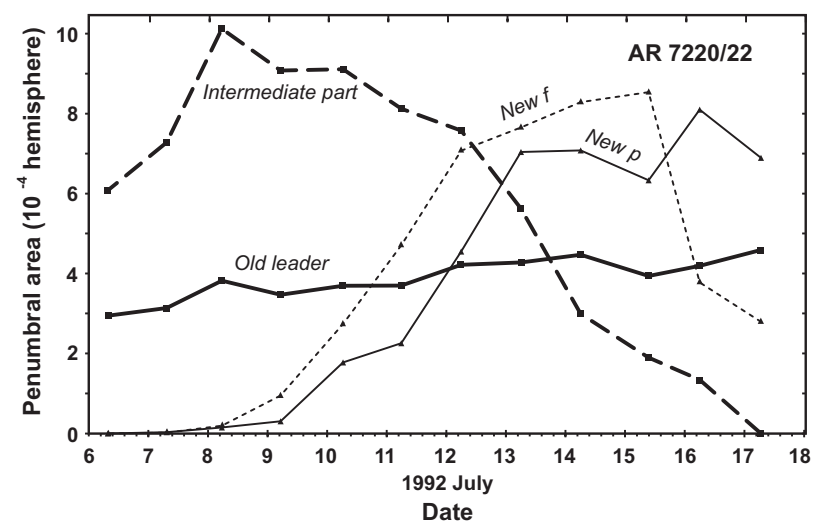

Fig. 5. Evolution of the total area of various parts of AR $7220 / 22$

opposite magnetic polaritites. The leader multi-umbra spot formed on July 14 (Fig. 3a), which interacted only with the $f$-part of the group (Fig. 7b). Motions in AR 7222 were characteristic for young bipolar groups: $p$-polarity westward, $f$-polarity eastward; only the small $f$-polarity umbrae north of the centre of the group moved significantly southeastward, moving nearer to the axis of the group. The $p$-polarity umbrae, as usual, were better defined and longer-lived, although very irregular. The umbrae in the $f$-part were more dispersed, and as the group approached the west limb they reduced strongly, so practically only the $p$-part with very elongated umbrae remained to return. AR 7222, although quickly growing and moving, was not very flare-active. From July 7 about 5-6 subflares of $\mathrm{C}$ class were observed on average. The only more active period was July 13-15, when emergence of spots was observed in the middle northern part of the group, (810 subflares and 1-2 Imp. 1 flares). The single $M$ class flare in this group was on July 14. After this period, with the beginning of the decay of the smaller spots in the $f$ part, the flare activity again subsided.

In the next rotation AR 7248 appeared in the same place on the solar surface. Although seemingly bipolar, in reality the umbrae were the $p$-polarity leaders of AR 7220 and 7222. Some pores and small spots evolved around

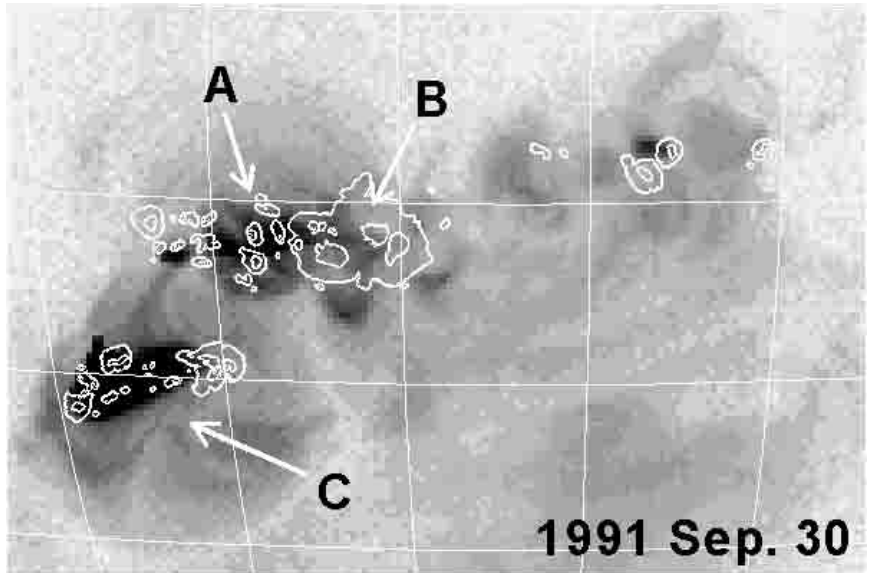

Fig. 6. Penumra and umbra contours of NOAA 6850 at 1991 Sep. 30 from Debrecen measurements, superposed on a negative YOHKOH image. Labels indicate the bright (on the negative - dark) loops, connecting the two polarities of the newly emerging flux $(A)$, the significantly less bright loops connecting the old dipole $(B)$ and the very bright loop system of the quickly evolving new AR $6853(C)$. No connecting loops between the old and newly emerging field are seen

them, but AR 7248 was principially a quiet sunspot group (Fig. 3b).

\section{Magnetic flux submergence}

Magnetic flux changes were derived from Kitt Peak magnetograms. For the passage of AR 6850 (September 26October 6,1991 ) there is a magnetogram available every day, except on Sep. 27. A mask image was created on the base of the magnetograms, on which the area of each magnetogram was subdivided into several regions, containing e.g. the old and new $p$ - and f-polarity, the moat area, the adjacent opposite polarity areas due to perspective, etc. Magnetic flux was derived for each region, taking into account the geometric foreshortening of the area. Figure 4 shows the change of the magnetic flux of the main parts in the spotgroup: the old double leader umbrae, the old $f$ part, jammed between the backwards drifting old and forwards streaming new $p$-polarities, and the $p$ - and $f$-polarity part of the new activity. The decay of the old fields mirrors the emergence of the new flux, the $f$-polarity part especially declines steadily, losing about $1610^{21}$ maxwells during the transit. The old $p$-polarity leader umbra pair also decays; this process is less pronounced, amounting to only a $410^{21}$ maxwells reduction between the first and last measurement. As noted in Bumba et al. (1995), on Oct. 14 the positive and negative fluxes were approximately balanced, with a slight positive $(p-)$ predominance. This can also be seen; in Fig. 4 , the $p$ - and $f$-polarity fluxes run almost parallel for the whole transit. The predominance of the positive $(p)$ polarity appears in the less steep decrease of the old $p$-flux and in the levelling off and later decrease of the new f-flux after Oct. 4. About $2010^{21}$ maxwells magnetic flux of both polarities disappeared during the studied 10 days of the transit from the old spotgroup. 

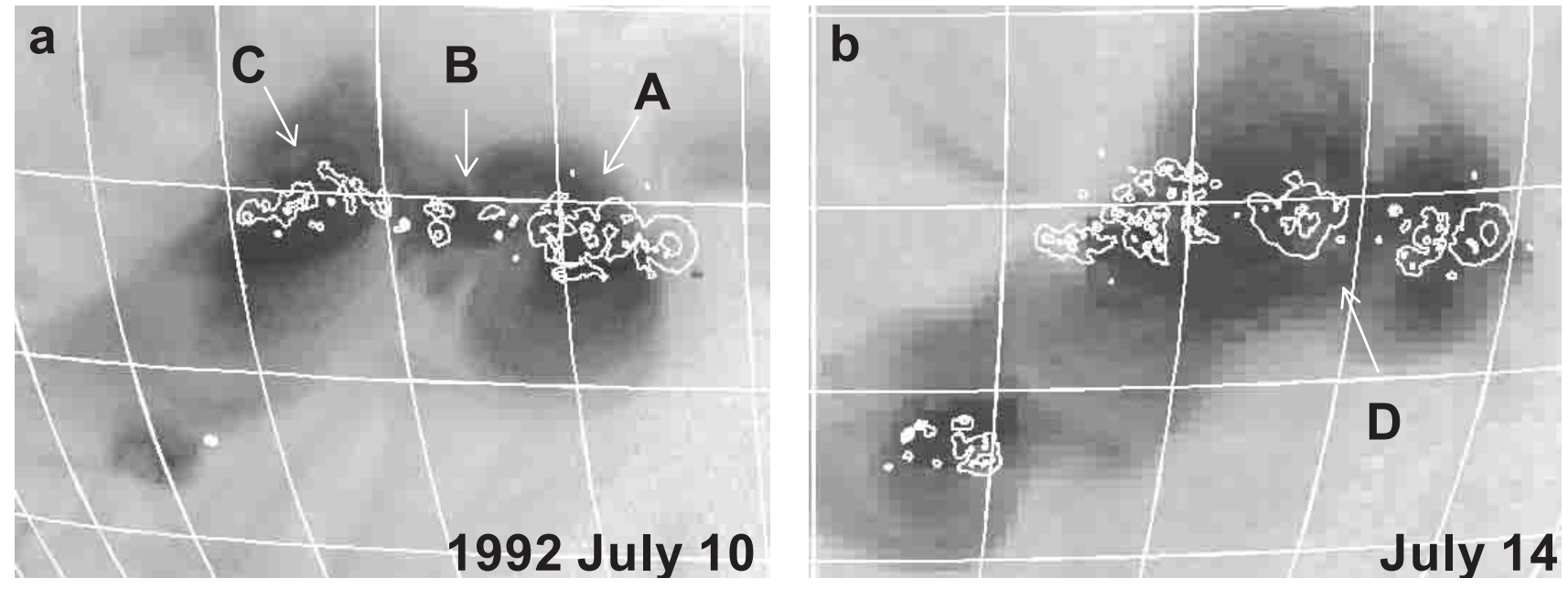

Fig. 7. Penumbra and umbra contours of NOAA 7220/22 at July 10 a) and 14 b), 1992, superposed on negative YOHKOH images. The three loop systems at July 10: $f$ - (but bipolar) part of AR $7220(A)$, dipoles between AR 7220 and $7222(B)$ and the quickly evolving bipolar part of AR $7222(C)$ are marked on a), as well as the gap at July 14 between the loops of AR 7220 and $7222(D)$ on $\mathbf{b})$

For AR 7220/22 only 5 KPNO daily magnetograms were available during 12 days; in Fig. 5 the change in penumbral areas of various parts are displayed: the old leader spot at the preceding (W) end of AR 7220, the emerging $p$ - and $f$-polarity parts of the new AR 7222, and the intermediate parts between these two. The first appearing $p$-polarity spots of AR 7222 , not showing westward drift during July 6-12 (Fig. 3a), are counted as members of the intermediate part, because these interacted with umbrae of opposite polarity in the $f$-part of AR 7220 (see Discussion). The old leader spot remained practically unchanged during the transit, with the umbral area slightly decreasing, and the penumbral area slightly increasing. The intermediate part began to decrease on July 8, mirroring the quick growth of the bipolar part of AR 7222. This intermediate part, containing both polarities, fully submerged on July 17, a 1000 millionths of solar hemisphere decay in 10 days. The new bipolar group continued to be balanced until July 15 ; the $f$-polarity part was even larger, but after that date the following part quickly dispersed and began to decay.

\section{Discussion and conclusions}

In the two examples shown, fairly significant amounts of magnetic flux disappeared in the interaction of old and new magnetic activity. In the case of AR 6850, mainly the $f$-polarity disappeared, in AR 7220/22 the whole bipolar spotgroup to the East of the leader umbra of 7220 submerged, including some of the newly emerged flux as well. Although in both cases there were colliding motions of different magnetic polarity umbrae, which led to fairly large magnetic gradients in AR 6850, no larger flares occurred in these sunspot groups. The collisions of the umbrae were head-on, so produced no shear, which seems to be needed for flare activity (Gaizauskas et al. 1994). Also, all of the interacting groups were simple, East-West (i.e. normally) oriented bipolar structures; the flare activity in NorthSouth oriented or especially in reverse (contrary to Hale's law) oriented bipolar groups is significantly higher.

The low flare activity of these spotgroups is also understandable from YOHKOH X-ray images. The coronal magnetic field, delineated by the loops, looks nearly potential, as the characteristic S-shape of the nonpotential fields (Sakurai et al. 1992; Canfield et al. 1999) is not seen anywhere. The emerging flux seemed more or less potential, and the photospheric motions did not disturb this state.

The newly emerging flux never interacted with the old one. In the case of AR 6850 on Sep. 30, a bright loop system connected the $p$ - and $f$-polarity parts of the newly emerging flux and less-bright loops could be seen between the old double leader umbra and the sinking $f$-polarity part, but no connection was visible between the old and the new flux (Fig. 6). For AR 7220/22, the leader spot of 7220 did not seem to have any bright loops in the X-ray images. An X-ray image of a C5.3 flare was published by Sylwester \& Sylwester (1996), where it is visible that the bright flare loops connect the umbrae of the compact bipolar part, but the large leader umbra does not participate in the activity. On July 10, bright loops connected only the $p$ - and $f$-polarities in the bipolar following part of AR 7220. There were also loops over the newly emerged intermediate dipoles between 7220 and 7222, and also over the main part of 7222 . This connection explains, why the newly appearing p-polarity umbrae of AR 7222 did not move regularly - they interacted with the new $f$-polarity spots on the eastern border of AR 7220 (Fig. 7a). As the forward-moving new $p$-polarity umbrae of AR 7222 grew and approached the remnant of the $f$-part of AR 7220 , their coronal connections remained connected to the $f$ part of 7222 and the field structures remained separate (Fig. 7b, this was shown also by Jiao et al. 1997). So the old, and some of the new, flux of both polarities between 
the leader spot of AR 7220 and 7222 simply submerged and disappeared.

The evolution of these active regions to the next rotation was significantly different. AR 6850 in the next rotation became AR 6891, a very complex, superactive region (Fig. 2b) with several white-light flares (Hudson et al. 1992). This was perhaps caused by the fact that only one magnetic polarity submerged, but more probably the activity was caused by the flow of newly emerging NorthSouth oriented bipolar flux from the eastern side of the passively behaving remnant $p$-polarity old leader umbrae, creating a wake with colliding opposite magnetic polarities on the western side of the old, stable multiple-umbra spot (Kálmán 1997).

ARs 7220 and 7222 evolved to AR 7248 to the next rotation (Fig. 3b). Only the leader spots remained, forming a quasi-bipolar group. The western spot, which was the former leader of AR 7220, was quiet also in this rotation, with no coronal loops. The eastern one, remnant of the more irregular (from the beginning) leader of AR 7222, was more active, both in the photosphere with emerging pores and small spots of both polarities around it, with associated smaller flares, and bright coronal loops. This activity was not as spectacular as that of AR 6891 . Seemingly, the submerged bipolar magnetic flux simply cancelled and disappeared in this case, as the new activity was not as close to the old one as in AR 6850, and also small intervening dipoles appeared in the process of the collision, preventing a closer subsurface interaction.

The disappearance of magnetic flux and sunspots was most probably due to submergence of the fluxtubes. No large flares were observed in these spotgroups, and as flares are thought to be connected with conversion and reconnection of the magnetic field, these processes could not play an important role. The turbulent erosion of the magnetic field of sunspots (Petrovay \& Moreno-Insertis 1997 ) is a slow process, unable to remove such a large flux in such a short time. The possibility of magnetic reconnection cannot be completely ruled out, however, because if it occurs in the photosphere (density $\sim 10^{23} \mathrm{~m}^{-3}$ ), the magnetic energy density is small compared to the thermal energy density of the photospheric plasma, so reconnection causes a temperature rise of only an unnoticeable few tens of degrees. So the appearance of new magnetic flux, new sunspots, and their interaction with the old ones, can lead to forced submergence and/or annihilation of the already existing photospheric magnetic flux, in these cases without significant flaring activity. This process can also be significant in the evolution of active regions. Gaizauskas et al. (1983) demonstrated, that in active complexes the magnetic flux is nearly constant, and is sustained by repeated emergence of new flux; at the same time the greater part of the flux disappears locally.

Acknowledgements. The photospheric observations in Debrecen were made by G. and J. Csepura, A. Kovács,
A. Ludmány and I. Nagy. Kitt Peak Observatory magnetograms were taken from the NSO/Kitt Peak Internet archives. NSO/Kitt Peak data used here are produced cooperatively by NSF/NOAO, NASA/GSFC and NOAA/SEL. The YOHKOH images were retrieved also through the Internet, from the NASA GSFC (USA) and MSSL (UK) Yohkoh Data Archive Centers. YOHKOH is a mission of the Japanese Institute for Space and Astronautical Science. The author wishes to thank V. Bumba for making available the Ondrejov observation of AR's 7220 and 7222 for July 13th, the many people who made the YOHKOH mission such a great success, J. Gurman from NASA GSFC for help in receiving the SXT data, also the anonoymous referee for directing attention to the possibility of low-level reconnection. This research has made use of NASA's Astrophysics Data System Abstract Service. Part of this work was supported by grant T-025737 of the Hungarian Scientific Research Fund (OTKA).

\section{References}

Bumba, V., Klvaňa, M., Kálmán, B., \& Györi, L. 1993, A\&A, 276, 193

Bumba, V., Klvaňa, M., \& Kálmán, B. 1995, A\&AS, 109, 355

Bumba, V., Klvaňa, M., Kálmán, B., \& Garcia, A. 1996, A\&AS, 117, 291

Canfield, R. C., Hudson, H. S., \& McKenzie, D. E. 1999, Geophys. Res. Lett., 26, 627

Cowling, T. G. 1946, MNRAS, 106, 218

Gaizauskas, V., Harvey, K. L., Harvey, J. W., \& Zwaan, C. 1983, ApJ, 265, 1056

Gaizauskas, V., Harvey, K. L., \& Proulx, M. 1994, ApJ, 422, 883

Garcia de la Rosa, J. I., \& Collados, M. 1987, in The Sun, ed. L. Hejna, \& M. Sobotka, Publ. Astr. Inst Czechosl. Acad. Sci., 1(66), 97

Harvey, K. L., Jones, H. P., Schrijver, C. J., \& Penn, M. J. 1991, Solar Phys., 190, 35

Hudson, H. S., Acton, L. W., Hirayama, H., \& Uchida, Y. 1992, PASJ, 44, L77

Jiao, L., McClymont, A. N., \& Mikić, Z. 1997, Solar Phys., 174,311

Kálmán, B. 1997, A\&A, 327, 779

Kunzel, H. 1960, Astron. Nachr., 285, 271

Ogawara, Y., et al. 1991, Solar Phys., 136, 1

Petrovay, K., \& Moreno-Insertis, F. 1997, ApJ, 485, 398

Priest, E., \& Forbes, T. 2000, Magnetic Reconnection (Cambridge University Press), $359 \mathrm{ff}$

Rabin, D., Moore, R., \& Hagyard, M. J. 1984, ApJ, 287, 404

Sakurai, T., Shibata, K., Ichimoto, K., Tsuneta, S., \& Acton, L. W. 1992, PASJ, 44, L123

Sammis, I., Tang, F., \& Zirin, H. 2000, ApJ, 540, 583

Sylwester, B., \& Sylwester, J. 1996, in JOSO Annual Report 1996, ed. M. Saniga, 97

Tsuneta, S., et al. 1991, Solar Phys., 136, 37

Wang, H. 1992, in The Solar Cycle, ed. K. L. Harvey, PASPC, 27,97

Zirin, H. 1985, ApJ, 291, 858

Zirin, H., \& Liggett, M. A. 1987, Solar Phys., 113, 267 